\title{
ESTIMATES OF HETEROSIS AND COMBINING ABILATY FOR FOLIAR DISEASES RESISTANCE, YIELD AND ITS COMPONENTES AND SEED QUILITY OF FABA BEAN
}

\author{
Salwa M. Mostafa(1), Gehan G. A. Abou-Zaid ${ }^{(1)}$, Shymaa F.A. kalboush ${ }^{(1)}$ \\ and Alaa M.E.A. Shahein ${ }^{(2)}$ \\ (1) Food Legumes Res. Sec. Field Crops Res. Inst., Agric. Res., Center (ARC), Egypt \\ ${ }^{(2)}$ Seed Tech. Res. Section, Field Crops Res. Inst., Agric. Res., Center (ARC), Egypt
}

Received: Dec. 20 , 2020

Accepted: Dec. 29,2020

ABSTRACT: Five diverse faba bean (Vicia faba L.) genotypes were crossed in half diallel method. The $F_{1}$ seeds along with their parental genotypes were sown in a randomized complete block design with three replications during 2019 /20 season at Sakha Agricultural Research Station. Analysis of variances revealed highly significant effects of genotypes for all the studied traits, providing evidence for the presence of large amount of genetic variability. The parental genotypes; Santamora and Sakha 3 were considered as good sources for resistance to chocolate spot and rust and high yield ability. Meanwhile, the parental genotypes; Giza 429 and $R \cdot V_{323}$ considered as good sources for early flowering. Due to the significance of gca and sca variances, the additive as well as non-additive components were more important for all the studied traits under study. The estimates of GCA/SCA mean squares were more than unity for all traits, except No. of branches, pods, seeds and crude protein content, where the same ratio was less than unity. This indicated that most of the genetic variation in these traits appear to be additive. Heterotic effects over mid and better parents were detected in the crosses. Based on the two estimates of heterotic effects, the following crosses: $C_{1}$ (Giza40 x Santamora), $C_{8}$ (Giza429 x Sakha3), $C_{9}$ (Giza429 x R.V) and C ${ }_{10}$ (Sakha3 x R.V) exhibited significant positive heterotic effects over both mid and better parents for most studied yield characters. Progenies of these crosses will be used in bulk method selection program to produce high yield potentiality pure lines.

Key words: Faba bean, Chocolate spot, Rust, Heterosis, Combining ability.

\section{INTRODUCTION}

Faba bean (Vicia faba L.) is widely considered as a good source of protein, starch and minerals for humans in developing countries and its content of cellulose is also useful for animals in industrialized countries (Haciseferogullari et al., 2003). In addition, faba bean is one of the most efficient fixers of the atmospheric nitrogen and, hence, can contribute to sustain or enhance total soil nitrogen fertility through bidogical $\mathbf{N}_{2}$-fixation (Lindemann and Glover, 2003).

Faba bean is a self-pollinate plant with significant levels of out-cross and inter- cross, ranging from 20 to $80 \%$ (Suso and Moreno, 1999) depending on genotype and environmental effects. The improvement of crop desired traits depends on the nature and magnitude of genetic variability and interactions involved in the inheritance of these traits which can be estimated using diallel cross technique. This technique may also result in the production of new genetic combinations whose performance, negatively or positively, may exceed that of the parents, a phenomenon known as heterosis. Exploitation of heterosis could pay off improving yield potential and its components in faba beans, where superiority of hybrids over the mid and/ 
Salwa M. Mostafa, et al.,

or better parents for seed yield is associated with manifestation of heterotic effects in important yield components, i.e., No. of branches per plant, No. of pods per plant and seed index. These heterotic effects may range from significantly negative to significantly positive for different traits depending on genetic make-up of parents (El-Hosary et al., 1997, Darwish et al., 2005, El-Hady et al., 2006 and Abou-Zaid et al., 2018).

The improvement of various traits depends on the nature and magnitude of genetic variability in addition to hybridization which offers new recombinations and release new materials for improvement and helps the breeders to identify the best combinations to be crossed either to exploit heterosis or build up the favorable fixable genes. Therefore, yield itself may not be the best criterion for selection, so that breeding for high seed yield is associated with yield and its components; No. of branches, pods, No. of seeds plant $^{-1}$ and 100 -seed weight (Rowlands, 1955).

Faba bean is widely considered as good source of protein, starch and minerals for humans and animals in industrialized countries (Haciseferogullari et al., 2003). Generally research on seed quality of faba bean, has been focused on total protein and carbohydrate (Tewatia and virk, 1996). Protein content which ranges from (27 to $34 \%$ depend on genotypes and carbohydrate content ranges between (52.3 to $64.4 \%$ ) on dry weight (Salih and El- Hardallou, 1986).

The present study aimed at determining the magnitude of heterosis, general and specific combining ability of some faba bean hybrid combinations.

\section{MATERIALS AND METHODS}

Five faba bean varieties, i.e., Giza 40 $\left(P_{1}\right)$, Santamora $1\left(P_{2}\right)$, Giza $429\left(P_{3}\right)$, Sakha $3\left(P_{4}\right)$ and $R . V\left(P_{5}\right)$ were selected on the basis of the presence of wide differences among them with respect to certain economically important traits and their reaction with the foliar diseases. The second and the fourth genotypes possess variable degrees of resistance to foliar diseases (chocolate spot and rust) while another genotypes are susceptible one as shown in Table 1.

Table 1: Names, origin', botanical group, disease reactions and agronomic characters of the five parental faba bean genotypes used in this investigation.

\begin{tabular}{|c|c|c|c|c|c|}
\hline \multirow[b]{2}{*}{ Genotypes } & \multirow[b]{2}{*}{ Origin } & \multirow{2}{*}{$\begin{array}{c}\text { Botanical } \\
\text { group }\end{array}$} & \multicolumn{3}{|c|}{ Agronomic characters } \\
\hline & & & $\begin{array}{l}\text { Disease } \\
\text { reactions }\end{array}$ & $\begin{array}{c}\text { Flowering } \\
\text { date }\end{array}$ & $\begin{array}{c}\text { Yielding } \\
\text { level }\end{array}$ \\
\hline Giza 40 & Egypt & Equina & $\mathbf{S}$ & Early & High \\
\hline Santamora & Spain & Major & $\mathbf{R}$ & Medium & High \\
\hline Giza 429 & Egypt & Equina & $\mathbf{S}$ & Early & High \\
\hline Sakha 3 & Egypt & Equina & $\mathbf{R}$ & late & High \\
\hline R. $\mathbf{V}_{323}$ & Sudan & Minor & $\mathbf{S}$ & Early & low \\
\hline
\end{tabular}

HR=High resistance to foliar diseases

MS= Moderate susceptibility to foliar diseases
$\mathbf{R}=$ Resistant to foliar diseases

$\mathrm{S}=$ Susceptibility to foliar diseases 
In 2018/2019 season under free insect cage, all possible cross combinations excluding reciprocals (half diallel) were made among the five parents (sown in two sowing dates to avoid differences in flowering times and to secure enough hybrids seeds during this season). Parents and derived $10 F_{1}$ 's were grown under the free insect cages at Sakha Agricultural Research Station Farm, Kafr El-Sheikh, Egypt during the season of 2019/2020. Fifteen genotypes were sown in a randomized complete block design with three replications under natural infection condition, surrounded by a highly foliar diseases infection variety. Seeds were sown in single seeded hills, $20 \mathrm{~cm}$ apart, each entry was represented by one row for parents and their $F_{1}$, s. The row was 3 meters long and $60 \mathrm{~cm}$ in between.
Measurements were taken on the basis of individual plant as follows:

Chocolate spot and rust disease reactions, flowering date, plant height, No. of branches plant ${ }^{-1}$, No. of pods plant $^{-1}$, No. of seeds plant ${ }^{-1}, 100$-seed weight, seed yield plant ${ }^{-1}$, crude protein $\%$ and carbohydrate \%.

The choice of parents was based on: a) genetic diversity. b) differences in growth habit and disease reactions and c) differences in yielding ability. The pedigree, disease reactions, agronomic characters and yielding level are presented in Table 1.

The disease severity of chocolate spot and rust diseases was recorded at midFebruary and mid-March, respectively using the scale of Bernier et al. (1984), as shown in Table 2.

Table 2: Chocolate spot and rust diseases scale.

\begin{tabular}{|l|l|}
\hline & Chocolate spot \\
\hline 3 & No disease symptoms or very small specks (highly resistance) \\
\hline 5 & Few small disease lesions (resistant) \\
\hline 7 & $\begin{array}{l}\text { Large coalesced sporulating lesions, } 50 \% \text { defoliation and some dead plants } \\
\text { (susceptible) }\end{array}$ \\
\hline 9 & $\begin{array}{l}\text { Extensive, heavy sporulation, stem girdling, blackening and death of more than } \\
80 \% \text { of plants (highly susceptible) }\end{array}$ \\
\hline 1 & No pustules or very small non-sporulating flecks (highly resistant) \\
\hline 3 & $\begin{array}{l}\text { Few scattered pustules covering less than } 1 \% \text { of the leaf area and few or no } \\
\text { pustules on stem (resistant) }\end{array}$ \\
\hline 5 & $\begin{array}{l}\text { Pustules common on leaves covering 1-4\% of leaf area, little defoliation and } \\
\text { some pustules on stem (moderately resistant) }\end{array}$ \\
\hline 7 & $\begin{array}{l}\text { Pustules very common on leaves covering 4-8\% of leaf area, some defoliation } \\
\text { and many pustules on stem (susceptible) }\end{array}$ \\
\hline Extensive pustules on leave, petioles and stem covering $8-10 \%$ of leaf area, many \\
\hline
\end{tabular}


Salwa M. Mostafa, et al.,

\section{Seed quality:}

Some seed properties were carried out at Sakha Seed Technology Research Department as follow :

Chemical composition including crude protein and carbohydrate content were determined according to the methods described in the AOAC (2006).

Mean squares and expected mean square of RCBD analysis of variance are presented in Table 3.

Where: $r$ is the number of replication; $E$ is the number of entries; $\sigma^{2} E$ and $\sigma^{2} e$ refer to genotypic and error variance, respectively. The difference between any two means was tested according to the least significant difference (LSD) at both $5 \%$ and $1 \%$ levels of significance as follows:

LSD : $P \leq 0.05=t 0.05$ (d.f) $x S_{d}$

$P<0.01=t 0.01$ (d.f) $\times S$ d

Where: $r$ is the number of replications and $\mathrm{Ms}_{\mathrm{e}}$ : is the mean squares of error

\section{Estimation of combining ability analysis:}

The sum of squares among entries (genotypes) is in turn partitioned into parents and crosses and the latest is partitioned into general combining ability (GCA) and specific combining ability (SCA) and $t$ is the tableted $t$ at the degrees of freedom of error. The combining ability analysis of variance and the expectation of mean squares are given in Table (3) according to model 1 method 2 of Griffing approach (1956). The effects of parental varieties and crosses were considered as fixed effects.

The mathematical model for the combining ability analysis is assumed to be:

$X i j k=u+\hat{g} i+\hat{g} j+\hat{S} \mathbf{i j}+r k+e i j k$

where: $x i j$, is the performance of the $i$ th parent mated to the $j^{\text {th }}$ parent in block $k$. $u$ is the population mean, $\hat{g} i$ is the gca effect of the $i^{\text {th }}$ parental variety, $\hat{\mathbf{S}} \mathrm{ij}$ is the interaction of the $i^{\text {th }}$ and $j^{\text {th }}$ parents or sca effect of the crosses between them $r$ $k$ is the block effect and eijk is the random efect of the indvidual observation.

The restrictions: $\Sigma \mathrm{i}(\hat{\mathbf{g}})=\mathbf{0}$ and $\Sigma \mathrm{j}\left(\hat{\mathrm{S}}_{\mathrm{ij}}+\hat{\mathrm{S}}_{\mathrm{ii}}\right)=$ 0 (for each $i$ ) are imposed on the combining ability effects.

\section{Heterosis:}

Heterosis was determined as outlined by Foolad and Bassiri (1983). Appropriate $t$-test was made for the significance of the $F_{1}$ 's from the mid and better parent (heterobeltiosis superiority of $F_{1}$ hybrids over the best parent) values (Wynne et al., 1970).

Table 3: The analysis of variance and the expected mean of square (EMS)

\begin{tabular}{|c|c|c|c|}
\hline S.O.V & $d \mathrm{f}$ & $M S$ & EMS \\
\hline Replication & $\mathrm{r}-1$ & $M \mathrm{r}$ & \\
\hline Genotypes & $(\mathrm{E}-1)$ & $M E$ & $\sigma^{2} \mathrm{e}+\mathrm{r} \sigma^{2} \mathrm{~g}$ \\
\hline G.C.A & $\mathrm{P}-1$ & $M g$ & $\sigma^{2} \mathrm{e}+(\mathrm{p}+1)(1 / \mathrm{p}-1) \Sigma_{\mathrm{gi}}$ \\
\hline S.C.A & $\mathrm{P}(\mathrm{P}-1) / 2$ & $M s$ & $\sigma^{2} \mathrm{e}+2 / \mathrm{p}(\mathrm{p}-1) \Sigma_{\mathrm{i}} \Sigma_{\mathrm{j}} \mathbf{s}_{\mathrm{ij}}$ \\
\hline Error & $(\mathrm{r}-1)(\mathrm{E}-1)$ & $M S \mathrm{e}$ & $\sigma^{2} \mathrm{e}$ \\
\hline Error term & $(\mathrm{r}-1)(\mathrm{E}-1) / 3$ & $M e$ & $\sigma^{2} \mathrm{e} / \mathrm{r}$ \\
\hline
\end{tabular}


The amount of heterosis was expressed as the percentage deviation of $F_{1}$ mean performance from the mid parent and better parent as follows:

Heterosis over mid - parent \% (M.P) =

$$
\left(\overline{\mathrm{F}}_{1}-\overline{\mathrm{MP}}\right) / \overline{\mathrm{MP}} \times 100
$$

Heterosis over better - parent \% (B.P) =

$$
\left(\bar{F}_{1}-\overline{B P}\right) / \overline{B P} \times 100
$$

LSD for mid-parent $\left(\bar{F}_{1}-\bar{M} \cdot P\right)=t(3 M S e / 2 r)^{1 / 2}$

LSD for better-parent $\left(\bar{F}_{1}-\bar{B} \cdot P\right)=t(2 M S e / r)^{1 / 2}$

\section{Potence ratio:}

This parameter was calculated according to Wigan(1944) and Mather and Jinks (1971) as follows:

$P . R=\bar{F}_{1}-\overline{M P} / 1 / 2(H P-L P)$

Where: $F_{1}=$ Mean of the $F_{1}$ performance.

$M . P=$ Mid-parent value $=P_{1}+P_{2} / 2$.

H.p = The hiegher parent value.

L. $\mathbf{P}=$ The lower parent value.

Absence of dominance is consider when $(p)$ is zero, and partial dominanance is assumed when $(p)$ is between less than +1 and more than -1 but not equal zero, complete dominance is considered when $(P)$ is equal +1 or -1 and over-dominance is considered when $(P)$ is $>+1$ or $<-1$.

\section{RESULTS AND DISCUSSION}

The analysis of variance for all studied traits are presented in Table 4. The results revealed that, mean squares due to genotypes were highly significant for all the studied traits, providing evidence for presence of large amount of genetic variability, which is considered adequate for further biometrical assessment. In addition, mean squares of GCA and SCA were significant and highly significant for all the studied traits.

The significance of GCA and SCA indicates the presence of both additive and non-additive gene effects in the genetic system controlling these traits. The mean squares of GCA/SCA ratio were more than one for chocolate and rust disease reactions, flowering date, plant height, seed yield plant $^{-1}$ and 100seed weight, indicating that additive type of gene effects play the major role in the inheritance of these traits. However, the same ratio was less than one for No. of branches plant $^{-1}$, No. of pods and seeds plant $^{-1}$, crude protein and carbohydrate (\%), may indicat that non-additive genes were responsible for the inheritance of these traits. These results confirmed those findings reported by Darwish et al., (2005), Attia and Salem (2006), El-Hady et al., (2007), Ibrahim (2012), Ghareeb and Helal (2014), Abdalla et al., (2017) and Abou-Zaid et al., (2018).

Table 4: Analysis of variance for yield and its components of faba bean in the $F_{1}$ generation.

\begin{tabular}{|l|c|c|c|c|c|c|}
\hline S.O.V & df & $\begin{array}{c}\text { Chocolate } \\
\text { spot disease } \\
\text { reaction }\end{array}$ & $\begin{array}{c}\text { Rust } \\
\text { disease } \\
\text { reaction }\end{array}$ & $\begin{array}{c}\text { Flowering } \\
\text { date (day) }\end{array}$ & $\begin{array}{c}\text { Plant } \\
\text { height (cm) }\end{array}$ & $\begin{array}{c}\text { No. of } \\
\text { branches } \\
\text { plant }\end{array}$ \\
\hline Reps & 2 & 0.11 & 0.07 & 1.7 & 3.77 & 0.06 \\
\hline Genotypes & 14 & $2.13^{\star \star}$ & $3.00^{\star \star}$ & $135.62^{\star *}$ & $756.28^{\star \star}$ & $2.75^{\star \star}$ \\
\hline GCA & 4 & $1.50^{\star \star}$ & $2.23^{\star \star}$ & $97.32^{\star \star}$ & $535.06^{\star \star}$ & $0.63^{\star \star}$ \\
\hline SCA & 10 & $0.39^{\star \star}$ & $0.51^{\star \star}$ & $24.36^{\star \star}$ & $138.91^{\star \star}$ & $1.03^{\star \star}$ \\
\hline Error & 24 & 0.14 & 0.11 & 1.82 & 9.26 & 0.32 \\
\hline Error term & & 0.05 & 0.04 & 0.61 & 3.09 & 0.11 \\
\hline GCA/SCA & & 3.84 & 4.37 & 3.99 & 3.85 & 0.61 \\
\hline
\end{tabular}

${ }^{\star}$ and ${ }^{\star *}$ significant at 0.05 and 0.01 levels of probability, respectively 
Salwa M. Mostafa, et al.,

Table 4: Cont.

\begin{tabular}{|l|c|c|c|c|c|c|c|}
\hline S.O.V & df & $\begin{array}{c}\text { No. of } \\
\text { pods } \\
\text { plant }^{-1}\end{array}$ & $\begin{array}{c}\text { No. of } \\
\text { seeds }_{\text {plant }^{-1}}\end{array}$ & $\begin{array}{c}\text { Seed yield } \\
\text { plant }^{-1} \\
(\mathrm{gm})\end{array}$ & $\begin{array}{c}100-\text {-seed } \\
\text { weight } \\
(\mathrm{gm})\end{array}$ & $\begin{array}{c}\text { crude } \\
\text { protein } \%\end{array}$ & $\begin{array}{c}\text { Carbohydrate } \\
\%\end{array}$ \\
\hline Reps & 2 & 3.81 & 18.91 & 6.73 & 0.24 & 0.38 & 0.42 \\
\hline Genotypes & 14 & $73.47^{\star \star}$ & $868.05^{\star \star}$ & $839.19^{\star \star}$ & $486.19^{\star \star}$ & $11.98^{\star \star}$ & $14.12^{\star \star}$ \\
\hline GCA & 4 & $3.76^{\star}$ & $93.70^{\star \star}$ & $352.12^{\star \star}$ & $427.01^{\star *}$ & $3.11^{\star \star}$ & $4.51^{\star \star}$ \\
\hline SCA & 10 & $32.78^{\star \star}$ & $367.61^{\star \star}$ & $250.78^{\star \star}$ & $56.09^{\star \star}$ & $4.35^{\star \star}$ & $4.79^{\star \star}$ \\
\hline Error & 24 & 4.04 & 17.71 & 8.71 & 2.83 & 0.45 & 0.48 \\
\hline Error term & & 1.35 & 5.9 & 2.9 & 0.94 & 0.15 & 0.16 \\
\hline GCA/ SCA & & 0.11 & 0.25 & 1.40 & 7.61 & 0.71 & 0.94 \\
\hline
\end{tabular}

*and $^{\star \star}$ significant at0.05 and 0.01 levels of probability, respectively

\section{Mean performance:}

Mean performance of parental varieties and their $F_{1}$ 's for all the studied traits are presented in Table 5. Results revealed that, the relative ranking scores of tested parental genotypes in descending order for chocolate spot and rust diseases resistance were Santamora $\left(P_{2}\right)$ and Sakha3 $\left(P_{4}\right)$ as resistant genotypes with $(4.0$ and 3.33), respectively, while Giza $40\left(P_{1}\right)$, Giza 429 $\left(P_{3}\right)$ and R.V $\left(p_{5}\right)$ were susceptible with values (5.81 and 6.0), (5.81 and 6.0) and (5. 09 and 5.70), respectively. The absence of complete resistance and susceptibility suggests the involvement of polygenic system (Abo-El-Zahab et al., 1994). Crosses involving the highly resistant parents exhibited the highest levels of resistance, $C_{6}$ (Santamora $x$ Sakha3) with (2.86 and 3.33) followed by $\mathrm{C}_{3}$ (Giza40 x Sakha3) (2.7 and 3.5) in chocolate spot and rust, respectively. However, cross involving the susceptible parents $C_{2}$ (Giza40 x Giza429) showed the least level of resistance (5.56 and 5. 97), respectively.

Highly significant differences between genotypes were found for flowering date revealed that the means of the parental varieties; Giza 429 and R.V were the earliest varieties (41.46 and 38.18 day, respectively). On the other hand, the parental variety; Sakha3 was the latest variety (58.57 day), While, the crosses; C2 (Giza $40 \times$ Giza 429), C4 (Giza $40 \times$ R. $V_{323}$ ) and $C_{9}$ (Giza $429 \times R^{2} V_{323}$ ) were considered as the earliest crosses. On the other side, $C_{1}$ (Giza40 x Santamora) behaved as the latest cross.

The variety; Santamora was the heaviest parent for seed yield plant $^{-1}$ and 100 -seed weight $\left(80.79 \mathrm{~g} \mathrm{plant}^{-1}\right.$ and 94.08 g) followed by Sakha3 with values $\mathbf{7 0 . 1 0}$ $\mathrm{g} \mathrm{plant}^{-1}$ and $85.34 \mathrm{~g}$ ), while the parental variety; R.V was the lowest $(38.12 \mathrm{~g}$ and 38.27 ) for seed yield and 100 -seed weight respectively. On the other hand, the crosses; $C_{1}$ (Giza40 x Santamora), $C_{8}$ (Giza429 x Sakha3) and $C_{10}$ (Sakha3 $x$ R. $V_{323}$ ) were the highest crosses for seed yield plant $^{-1}(87.68,105.17$ and $91.45 \mathrm{~g}$, respectively). The crosses; $\mathrm{C}_{3}$ (Giza40 $\mathrm{x}$ Sakha3) and $C_{4} \quad\left(G i z a 40 \quad x \quad\right.$ R. $\left.V_{323}\right)$ performed as low yield crosses (57.15 and $57.78 \mathrm{~g}$, respectively). For crude protein \%, Santamora containing of $(27.45 \%)$ and R.V V23 $_{32}(27.75 \%)$ had the highest parental values of crude protein $\%$, while $\mathrm{C}_{4}$ (Giza $40 \times$ R. $\mathrm{V}_{323}$ ) and $\mathrm{C}_{6}$ (Santmora $X$ sakha 3 ) were the highest crosses (31.26 and $29.25 \%$, respectively). Giza 429 behaved as the highest parent for carbohydrate $(61.33 \%)$ followed by Sakha3 $(60.90 \%)$, also $C_{1}, C_{2}$ and $\mathrm{C}_{5}$ behaved as the high carbohydrate $\%$ content $(61.93,60.25$ and $60.59 \%$, respectively). 


\begin{tabular}{|c|c|c|c|c|c|c|c|c|c|c|c|c|c|c|c|c|c|}
\hline 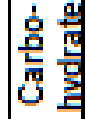 & స్ & ల్లై & 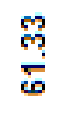 & $\begin{array}{l}\text { : } \\
\text { ○े }\end{array}$ & $\begin{array}{l}\text { ळ్ } \\
\text { డి }\end{array}$ & $\frac{\text { \% }}{5}$ & సุ & $\begin{array}{l}\stackrel{2}{2} \\
\stackrel{6}{\circ}\end{array}$ & 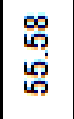 & 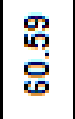 & 空 & 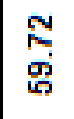 & 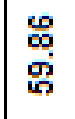 & 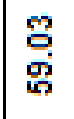 & \begin{tabular}{|l} 
జ \\
$\infty$ \\
$\infty$
\end{tabular} & $\tilde{\omega}$ & 옹 \\
\hline 号贯 & 㝏 & 㳯 & $\stackrel{\text { ஸ̃ }}{\vec{N}}$ & $\begin{array}{l}\text { œ } \\
\text { ని }\end{array}$ & 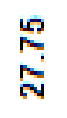 & $\begin{array}{l}\text { ల్లె } \\
\text { مై }\end{array}$ & 守 & $\begin{array}{l}\Theta \\
\stackrel{N}{N}\end{array}$ & 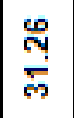 & $\begin{array}{l}\text { సొ } \\
\text { సి }\end{array}$ & 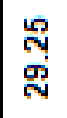 & 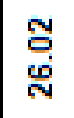 & $\begin{array}{l}\text { ๘ } \\
\text { స̃ }\end{array}$ & $\begin{array}{l}\text { 号 } \\
\text { స̃ }\end{array}$ & $\begin{array}{l}\text { \% } \\
\stackrel{9}{N} \\
\stackrel{N}{2}\end{array}$ & $\begin{array}{l}\mathscr{2} \\
0 \\
0\end{array}$ & $\begin{array}{l}\stackrel{\infty}{\infty} \\
\stackrel{0}{0}\end{array}$ \\
\hline 응 몀 & 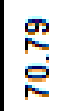 & $\begin{array}{l}\stackrel{\circ}{\circ} \\
\dot{\sigma}\end{array}$ & 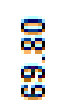 & 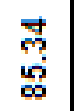 & 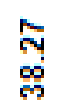 & 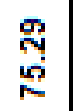 & 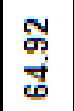 & $\stackrel{\text { ָ̊ }}{\stackrel{R}{R}}$ & 今ึ & $\underset{\text { S }}{\text { S }}$ & $\begin{array}{l}\stackrel{2}{2} \\
\stackrel{0}{\infty}\end{array}$ & $\begin{array}{l}\text { م } \\
\text { مू } \\
\text { م) }\end{array}$ & $\underset{\substack{R \\
\text { o } \\
r}}{R}$ & 艿 & 妴 & 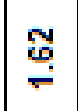 & $\frac{\text { D }}{\text { N }}$ \\
\hline 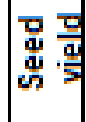 & $\begin{array}{l}\stackrel{0}{\circ} \\
\stackrel{0}{0}\end{array}$ & 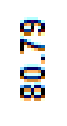 & フู่ & 웅 & $\underset{\infty}{\stackrel{\infty}{\infty}}$ & $\begin{array}{l}\infty \\
\stackrel{\infty}{\infty} \\
\end{array}$ & 웅 & $\frac{n}{5}$ & $\stackrel{\infty}{\stackrel{\infty}{\tilde{N}}}$ & $\stackrel{\mathscr{C}}{\stackrel{\Sigma}{R}}$ & $\begin{array}{l}\text { 怘 } \\
\text { ஸे }\end{array}$ & 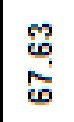 & 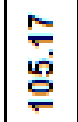 & 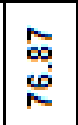 & 导 & $\begin{array}{l}\infty \\
\text { N } \\
\text { N }\end{array}$ & $\begin{array}{l}\vec{\Phi} \\
\text { ले }\end{array}$ \\
\hline 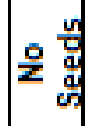 & సุ & $\begin{array}{l}\text { छే } \\
\text { 今 }\end{array}$ & స్ఠ & $\begin{array}{l}\text { ֻே } \\
\text { ஸ் }\end{array}$ & $\frac{\infty}{\stackrel{\infty}{\infty}}$ & $\begin{array}{l}\stackrel{\mathscr{\sigma}}{\circ} \\
\stackrel{=}{=}\end{array}$ & 品 & $\begin{array}{l}\text { జ్ } \\
\text { జ్ }\end{array}$ & స్ & $\begin{array}{l}\text { œ } \\
\stackrel{\sigma}{\sigma}\end{array}$ & $\begin{array}{l}\text { ల్ల } \\
\text { ๙ై }\end{array}$ & 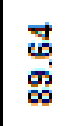 & 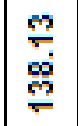 & $\begin{array}{l}\text { 오 } \\
\text { பे } \\
\text { 음 }\end{array}$ & స̃ & $\begin{array}{l}\mathscr{c} \\
\dot{\nabla}\end{array}$ & \ٌ \\
\hline 웜 & $\underset{\sim}{\approx}$ & $\underset{\sim}{\stackrel{\sim}{\sim}}$ & 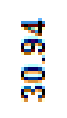 & 晶 & $\begin{array}{l}\stackrel{\infty}{\sim} \\
\stackrel{\omega}{\sim}\end{array}$ & ल्लై & 웅 & ث્也 & 萬 & 亏્ळ & 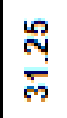 & 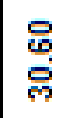 & ণั & 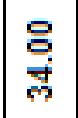 & 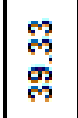 & $\stackrel{\vec{\sigma}}{-}$ & $\underset{\sim}{\text { ִָ }}$ \\
\hline 울 & స్ల & ָั & $\widehat{\widehat{\varphi}}$ & $\underset{\text { ?ु }}{\text { ?ु }}$ & $\begin{array}{l}\text { § } \\
\text { ๓ं }\end{array}$ & 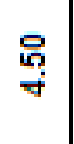 & 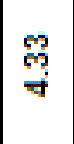 & $\hat{\ddot{\varphi}}$ & $\begin{array}{l}\hat{\omega} \\
\stackrel{n}{ }\end{array}$ & $\underset{\nabla}{ت}$ & $\begin{array}{l}\stackrel{g}{6} \\
\text { ल }\end{array}$ & 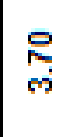 & $\begin{array}{l}\text { ஜ } \\
\text { ஸ }\end{array}$ & $\underset{\text { 筞 }}{\mathrm{s}}$ & $\begin{array}{l}\stackrel{\mathscr{2}}{\sim} \\
\boldsymbol{\omega}\end{array}$ & 芯 & $\underset{\overbrace{}}{\stackrel{0}{0}}$ \\
\hline 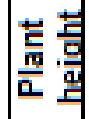 & 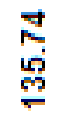 & 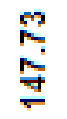 & 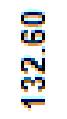 & $\begin{array}{l}\circ \\
\stackrel{0}{\circ} \\
\stackrel{\circ}{\circ}\end{array}$ & 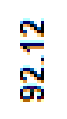 & 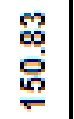 & 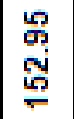 & $\begin{array}{l}\tilde{\omega} \\
\dot{\omega} \\
\stackrel{m}{-}\end{array}$ & 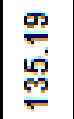 & $\begin{array}{l}\mathcal{N} \\
\stackrel{9}{-} \\
\stackrel{5}{-}\end{array}$ & 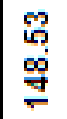 & $\begin{array}{l}\text { 号 } \\
\text { 主 }\end{array}$ & $\begin{array}{l}\text { 2̦ } \\
\stackrel{5}{2}\end{array}$ & $\begin{array}{l}\text { q } \\
\text { qें } \\
\text { pे }\end{array}$ & 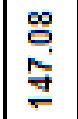 & 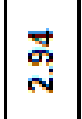 & $\begin{array}{l}\mathscr{\sigma} \\
\stackrel{m}{m}\end{array}$ \\
\hline 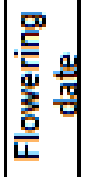 & $\underset{\sim}{\stackrel{\sim}{\sim}}$ & $\frac{\infty}{\text { ల్ర }}$ & $\stackrel{\mathscr{f}}{\dot{y}}$ & 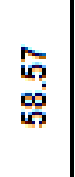 & $\underset{\infty}{\infty}$ & จै & 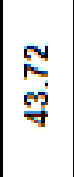 & $\begin{array}{l}\bar{\infty} \\
\dot{J}\end{array}$ & $\frac{\text { 20 }}{\text { ș }}$ & $\begin{array}{l}\stackrel{\infty}{\tilde{m}} \\
\text { ษ }\end{array}$ & ڤே & ஜு & 㚇 & $\begin{array}{l}\text { 号 } \\
\text { \% }\end{array}$ & 它 & 吊 & 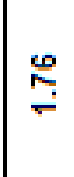 \\
\hline 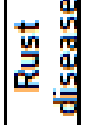 & $\stackrel{8}{\circ}$ & लె & $\underset{\text { ¿ }}{\circ}$ & ભ్లె & gi & 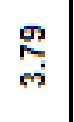 & $\begin{array}{l}\mathscr{\omega} \\
\omega \\
\omega\end{array}$ & $\underset{m}{\bar{m}}$ & สิ & ๙్ & $\begin{array}{l}\mathscr{D} \\
\stackrel{N}{N}\end{array}$ & શ્ల & $\frac{m}{i}$ & $\underset{+}{\circ}$ & $\begin{array}{l}\hat{\varphi} \\
\dot{\nabla}\end{array}$ & ల్ల & 导 \\
\hline 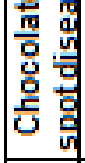 & $\begin{array}{c}\bar{\infty} \\
\text { ம. } \\
\end{array}$ & $\begin{array}{l}\text { 우 } \\
\dot{\forall}\end{array}$ & $\begin{array}{l}\bar{\infty} \\
0 \\
0\end{array}$ & $\underset{\forall}{\stackrel{\circ}{0}}$ & $\underset{\text { in }}{\stackrel{P}{0}}$ & $\frac{m}{6}$ & 寅 & $\widehat{\widehat{\omega}}$ & $\underset{\forall}{J}$ & $\stackrel{\sigma}{\underset{\nabla}{+}}$ & 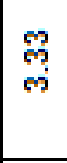 & $\begin{array}{l}\tilde{\varphi} \\
\dot{\nabla}\end{array}$ & $\begin{array}{l}\widehat{e} \\
\dot{\forall}\end{array}$ & ○ั & m & 吕 & ষ্? \\
\hline 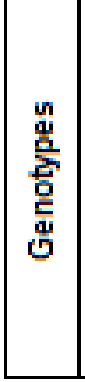 & 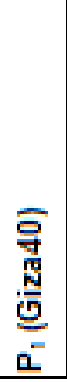 & 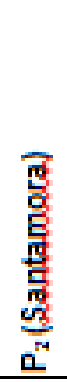 & 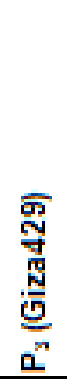 & 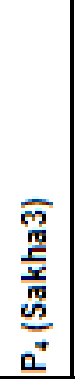 & 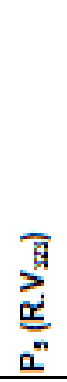 & 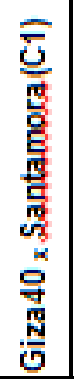 & 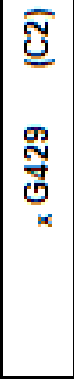 & $\begin{array}{l}\widehat{\widetilde{g}} \\
m \\
m \\
\frac{m}{5} \\
m \\
w \\
w\end{array}$ & 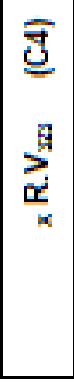 & 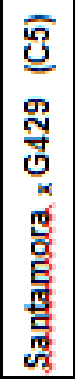 & 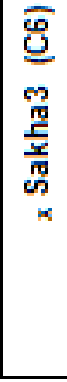 & 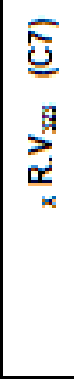 & 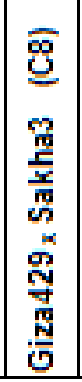 & 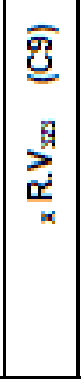 & 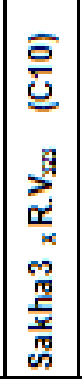 & 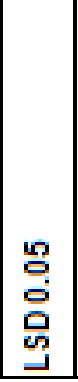 & $\begin{array}{l}5 \\
\text { 웅 } \\
\text { 웅 }\end{array}$ \\
\hline
\end{tabular}




\section{Combining ability:}

The estimates of GCA effects (ĝi) listed in Table 6, where differed from one individual parent to another and from trait to trait. The parental genotypes Santamora $\left(P_{2}\right)$ had highly significant negative (favorable) (ĝi) for chocolate spot and rust with -1.48 and -0.60 , respectively and high significance positive (ĝi) for plant height (5.48), seed yield plant ${ }^{-1}(6.19)$ and 100 seed weight (9.07). Giza429 $\left(P_{3}\right)$ had highly significant negative (favorable) (ĝi) for flowering date $(-3.73)$ and high significant positive (ĝi) for No. of pods (1.0), No. of seeds plant $^{-1}(3.91)$, seed yield plant ${ }^{-1}(1.54)$ and carbohydrate \% (1.29). Sakha3 $\left(p_{4}\right)$ had highly significant negative (favorable) (ĝi) effects for chocolate spot and rust with 0.53 and -0.61 respectively and high significance positive (ĝi) for plant height (8.63), No. of branches (0.27), No. of seed plant-1 (3.02) seed yield plant ${ }^{-1}$ (7.05), 100 seed weight (5.89) and crude protein \% (0.37). R. $V_{323}\left(p_{5}\right)$ had highly significant negative (favorable) (ĝi) for flowering date $(-3.60)$ and high significant positive (ĝi) for crude protein \% (0.85). H0wever, the parental genotypes; Sakha 3 had significant (ĝi) values in favorable direction for eight traits out of eleven ones and Santamora and Giza 429 had significant (ĝi) values for five out of eleven traits in favorable directions which may indicate that, these parents behaved as good combiners for the traits in question in the environmental condition of the present study. Therefore, these parents are favorable for inclusion in the production of synthetic varieties and choosing the roper breeding scheme. Similar trend of these findings was earlier reported by Drwish et al., (2005), El-Hady et al (2007and 2008), El-Bramawy and Osman (2012) and Abou-Zaid et al., (2018).

Table 6: Estimates of parental general combining ability effects for yield and its components, Carbohydrates and Crude Protein content (in the $F_{1}$ generation).

\begin{tabular}{|c|c|c|c|c|c|c|c|c|c|c|c|}
\hline Genotypes & $\begin{array}{c}\text { Chocolate } \\
\text { spot } \\
\text { disease } \\
\text { reaction }\end{array}$ & $\begin{array}{c}\text { Rust } \\
\text { disease } \\
\text { reaction }\end{array}$ & $\begin{array}{c}\text { Flowering } \\
\text { date }\end{array}$ & $\begin{array}{c}\text { Plant } \\
\text { height }\end{array}$ & $\begin{array}{c}\text { No. of } \\
\text { branches } \\
\text { plant }^{-1}\end{array}$ & $\begin{array}{l}\text { No. of } \\
\text { Pods } \\
\text { plant }^{-1}\end{array}$ & $\mid \begin{array}{l}\text { No. of } \\
\text { Seeds } \\
\text { plant }^{-1}\end{array}$ & $\mid \begin{array}{c}\text { Seed } \\
\text { yield } \\
\text { plant }^{-1}\end{array}$ & $\begin{array}{c}100-\text { seed } \\
\text { weight }\end{array}$ & $\begin{array}{c}\text { crude } \\
\text { protein } \\
\text { content }\end{array}$ & $\begin{array}{c}\text { Carbohy } \\
\text { drate } \\
\%\end{array}$ \\
\hline P1 (Giza40) & $0.31^{\star \star}$ & $0.42^{\star \star}$ & -0.21 & 0.57 & -0.22 & -0.38 & $4.79 *$ & $-6.45^{\star \star}$ & $-2.88^{\star \star}$ & -0.05 & 0.10 \\
\hline P2(Santamora) & $-0.48^{\star \star}$ & $-0.60^{\star \star}$ & $3.36^{\star \star}$ & $5.48^{\star \star}$ & $-0.42^{\star \star}$ & -0.54 & $2.47^{*}$ & $6.19^{\star \star}$ & $9.07^{\star \star}$ & -0.25 & $-0.68^{\star \star}$ \\
\hline P3 (Giza429) & $0.40^{* *}$ & $0.55^{\star \star}$ & $-3.73^{\star \star}$ & -0.51 & 0.16 & $1.00^{*}$ & $3.91^{\star \star}$ & $\star 1.54^{\star}$ & $-1.26^{\star \star}$ & $-0.92^{\star *}$ & $1.29^{\star \star}$ \\
\hline P4 (Sakha3) & $-0.53^{\star *}$ & $-0.61^{\star \star}$ & $4.18^{\star \star}$ & $8.63^{\star *}$ & $0.27^{\star}$ & 0.56 & $3.02^{\star \star}$ & $\star 7.05^{\star \star}$ & $5.89^{\star \star}$ & $0.37^{\star *}$ & -0.05 \\
\hline$P_{5}\left(R . V_{323}\right)$ & $0.31^{* *}$ & $0.23^{\star \star}$ & $-3.60^{* *}$ & $-14.17^{\star *}$ & 0.21 & -0.64 & 0.33 & $-8.32^{\star *}$ & $-10.82^{\star *}$ & $0.85^{\star \star}$ & $-0.66^{\star \star}$ \\
\hline LSD 0.05 & 0.15 & 0.13 & 0.54 & 1.22 & 0.23 & 0.80 & 1.68 & 1.18 & 0.67 & 0.27 & 0.28 \\
\hline 0.01 & 0.20 & 0.18 & 0.73 & 1.64 & 0.30 & 1.08 & 2.27 & 1.59 & 0.91 & 0.36 & 0.37 \\
\hline
\end{tabular}

${ }^{\star}$ and ${ }^{\star \star}$ significant at 0.05 and 0.01 levels of probability, respectively 
The SCA effects (Ŝij) are presented in Table 7. Significant and highly significant negative (favorable) (Sij) for chocolate spot were observed for the crosses; $C_{3}, C_{4}, C_{5} C_{6}$ and $C_{9}$. Also, significant and highly significant negative (Ŝij) for rust were observed for the crosses; $C_{3}, C_{4}$ and $C_{9}$. For flowering date, highly significant negative (favorable) (Ŝij) were observed for the crosses; $C_{1}, C_{3}, C_{5}$ and $C_{6}$. While, the (Sij) for seed yield plant ${ }^{-1} a$ highly significant positive (Sij) were observed for the crosses; $C_{1}, C_{3}, C_{8}, C_{9}$ and $C 10$. For crude protein \% the crosses; $\mathrm{C}_{2}, \mathrm{C}_{4}, \mathrm{C}_{6}$ and $\mathrm{C}_{8}$ exhibited a highly significant positive (Sij). With respect to carbohydrate \% the crosses; $C_{1}, C_{8}$ and $C_{10}$ gave a highly significant positive (Ŝij). However, the cross; Sakha3 $\times R^{R} V_{323}$ had significant and/or highly significant(Ŝij) for seven traits out of eleven ones i.e., plant height, No. of branches plant $^{-1}$, No. of pods plant ${ }^{-1}$, No. of seeds plant ${ }^{-1}$, seed yield plant ${ }^{-1}, 100$-seed weight and carbohydrate \% ; the cross; Giza429 x Sakha3 had highly significant (Ŝij) for six traits i.e., No of branches plant ${ }^{-1}$, No, of pods plant-1, No of seeds plant -1 , seed yield plant ${ }^{-1}$,crude protein \% and carbohydrate \%; the cross; Giza40 x Santamora had highly significant (Ŝij) for five traits i.e., plant height, No of branches plant ${ }^{-1}$,No.of pods plant ${ }^{-1}$, No. of seeds plant $^{-1}$, seed yield plant-1 and carbohydrate \%.These crosses could be used with follow suitable breeding method in segregating generations to obtain same line (s) characterized by high yielding ability and high carbohydrate\%. On the other hand, the cross Giza40 x Sakha3 had highly significant (Sิij) for chocolate spot and rust diseases and flowering date; the crosses; Giza40 x R. $V_{323}$ and Giza429 $\times R . V_{323}$ had highly significant (Ŝij) for chocolate spot and rust diseases, therefore, it could be use the progenies of these crosses in the segregating generations to generate line (s)with high tolerate to these diseases.

Combining ability analysis helps the breeders to identify the best combiners which may be hybridized either to exploit heterosis or to build up the favorable fixable genes. GCA effects provide appropriate criterion for detecting the validity of a genotype in hybrid combinations. While SCA effects may be related to heterosis. The results revealed that GCA effects, for some traits, were related to several SCA values of their corresponding crosses. This may indicate, in such combinations, that additive and nonadditive genetic systems present in the crosses are acting in the same direction to maximize the characters in view. These findings are in agreement with Darwish, et al. (2005), Attia and Salem (2006) and El-Hady, et al. (2007 and 2008).

\section{Heterosis:}

The results in Table 8 showed that, the crosses; Giza 40 x Sakha 3, Giza 40 x R.V323 and Santa Mora x Sakha 3 had significant mid - parental heterotic effects in negative direction due to over-dominance, while partial dominance was responsible to the midparental heterosis in the cross Santamora $x$ Giza429. For rust disease reaction, the crosses; Giza $40 \times$ Sakha3 and Giza40 x R. $V_{323}$ had highly significant mid-parental heterosis in negative direction (favorable) due to over-dominance, while the crosses; and Santa mora x Sakha 3 and Giza429 x Sakha3 had highly significant mid-parental heterosis due to partial- dominance as potence ratio pointed out.

For flowering date, the crosses; Giza $40 \times$ Sakha3 and Giza429 x R. $V_{323}$ showed highly significant mid-parental heterosis in negative direction (favorable) due to overdominance and the cross; Santamora $x$ Giza429 expressed highly significant mid parental heterosis due to partial- dominance .The crosses ;Giza40 x Santamora and Giza40 xGiza429 had 


\begin{tabular}{|c|c|c|c|c|c|c|c|c|c|c|c|c|}
\hline 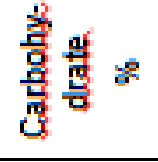 & $\stackrel{\frac{1}{0}}{\circ}$ & ஸุ & مீ & वै & సุ & ग्ञ & ஸ্ণ & $\stackrel{\text { }}{\stackrel{\text { }}{\circ}}$ & 号 & i⿱ & 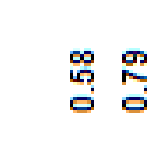 & 品 正 \\
\hline 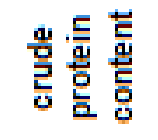 & $\frac{\stackrel{n}{\frac{1}{5}}}{\frac{1}{1}}$ & 势 & :ै & $\begin{array}{l}\text { 营 } \\
\stackrel{6}{0} \\
\text { ले }\end{array}$ & 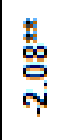 & लू & 芦 & $\stackrel{n}{n}$ & ల్లా & $\stackrel{\frac{1}{2}}{\frac{0}{0}}$ & 怘 & 웡 巳 \\
\hline 응 吾帚 & $\begin{array}{l}\text { t: } \\
\text { ò } \\
\dot{7}\end{array}$ & $\begin{array}{l}\text { t. } \\
\stackrel{4}{a} \\
\dot{7}\end{array}$ & 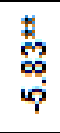 & 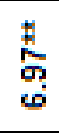 & 章 & $\stackrel{?}{?}$ & 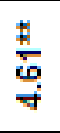 & 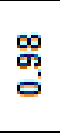 & 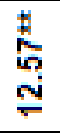 & సี๋ & 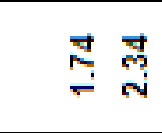 & 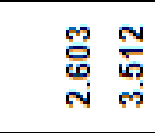 \\
\hline 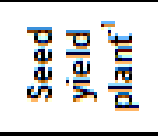 & 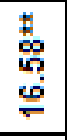 & $\begin{array}{l}\text { : } \\
\stackrel{0}{0} \\
\text { Tे } \\
\end{array}$ & $\begin{array}{l}\frac{1}{\infty} \\
\dot{\infty} \\
+ \\
\end{array}$ & $\stackrel{\infty}{\leftarrow}$ & 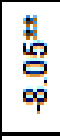 & $\stackrel{8}{\circ}$ & $\frac{\bar{\varphi}}{\square}$ & 范 & 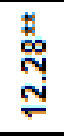 & 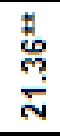 & 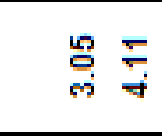 & 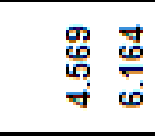 \\
\hline 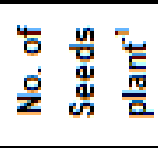 & $\begin{array}{l}\stackrel{ \pm}{\hat{n}} \\
\stackrel{\sim}{N}\end{array}$ & 总 & 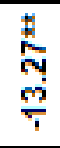 & 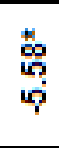 & $\frac{\varphi}{\dot{p}}$ & $\underset{T}{\stackrel{N}{T}}$ & \begin{tabular}{l}
$\stackrel{*}{*}$ \\
\multirow{2}{*}{} \\
\multirow{\rho}{*}{}
\end{tabular} & 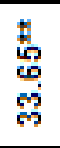 & $F_{\dot{m}}$ & 竞 & 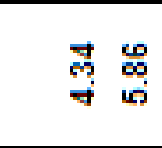 & 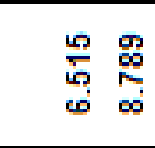 \\
\hline 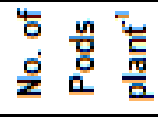 & 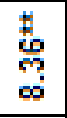 & $\stackrel{\varphi}{\leftarrow}$ & $\begin{array}{l}\text { ث̈. } \\
\text { î }\end{array}$ & $\stackrel{0}{+}$ & 함 & $\begin{array}{l}\ddot{0} \\
\ddot{9}\end{array}$ & $\frac{5}{9}$ & $\begin{array}{l}\stackrel{5}{2} \\
\text { N } \\
\end{array}$ & $\stackrel{2}{\stackrel{2}{\leftarrow}}$ & 藋 & స̊ & $\underset{\text { के }}{F} \frac{\hat{\sigma}}{\sigma}$ \\
\hline 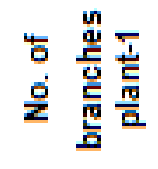 & $\stackrel{\frac{1}{6}}{-}$ & ஸุ & مீ & 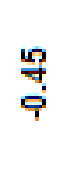 & ஸุ & 帘 & ণ্ডি & $\begin{array}{l}\text { है } \\
\text { क } \\
\stackrel{2}{\circ}\end{array}$ & : & $\frac{1}{\mathrm{n}}$ & ڤొ & $\underset{\substack{\mathfrak{\infty} \\
0}}{=}$ \\
\hline 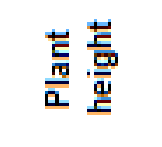 & : & $\begin{array}{l}\stackrel{\text { : }}{\mathrm{m}} \\
\stackrel{\mathrm{N}}{\sim}\end{array}$ & 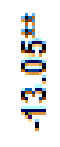 & 营 & 晜 & $\frac{1}{9}$ & $\frac{5}{5}$ & ஸ் & 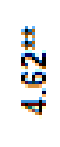 & $\stackrel{ }{\stackrel{ }{ }}$ & $\underset{\text { in }}{\vec{~}}$ & 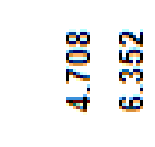 \\
\hline 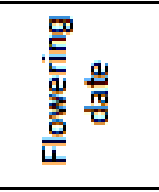 & $\begin{array}{l}\text { t. } \\
\text { ơ } \\
\text { oे }\end{array}$ & : & 芯 & ָָ & 范 & $\begin{array}{l}\text { : } \\
\text { : } \\
\dot{7}\end{array}$ & $\begin{array}{l}\text { th } \\
\infty \\
\infty \\
\text { ले }\end{array}$ & 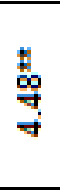 & $\stackrel{\circ}{\circ}$ & 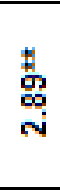 & $\stackrel{\text { g్ }}{-} \stackrel{\infty}{\leftarrow}$ & 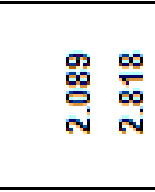 \\
\hline 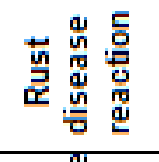 & ల్లా & ભొ & 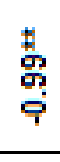 & స્ّ & 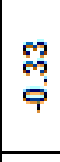 & ণ্ড় & ڤ్లై & 웅 & 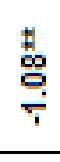 & 啙 & ભొ & 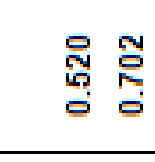 \\
\hline 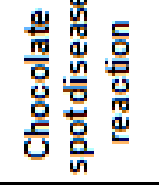 & 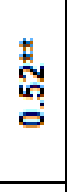 & 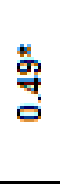 & 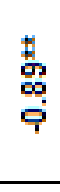 & 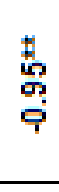 & 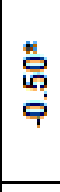 & सें & $\stackrel{\circ}{\circ}$ & 웅 & 苂 & 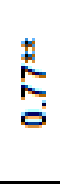 & 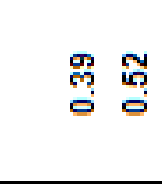 & 恕 \\
\hline 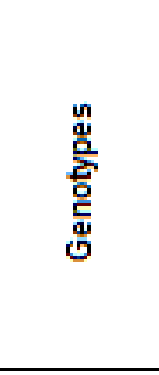 & 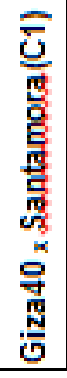 & 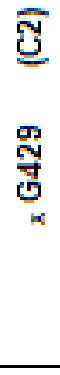 & 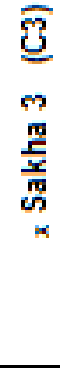 & 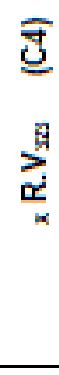 & 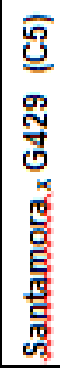 & 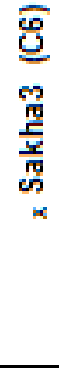 & 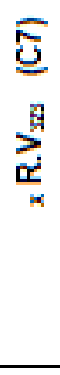 & 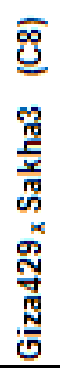 & 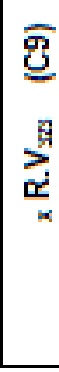 & 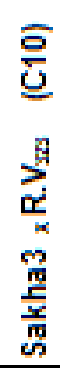 & 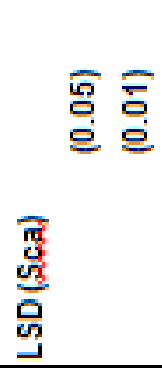 & 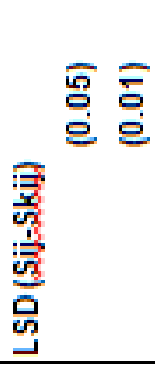 \\
\hline
\end{tabular}


Table 8: Heterotic effects relative to mid, better parent and potence ratios.

\begin{tabular}{|c|c|c|c|c|c|c|c|c|c|}
\hline \multirow{2}{*}{ Crosses } & \multicolumn{3}{|c|}{$\begin{array}{c}\text { Chocolate spot disease } \\
\text { reaction }\end{array}$} & \multicolumn{3}{|c|}{ Rust disease reaction } & \multicolumn{3}{|c|}{ Flowering date } \\
\hline & MP & PR & BP & MP & PR & BP & MP & PR & BP \\
\hline Giza40 $\times$ Santamora $(\mathrm{C} 1)$ & 4.43 & 0.23 & $28.13^{\star \star}$ & $-18.75^{\star \star}$ & \begin{tabular}{|l|}
-0.68 \\
\end{tabular} & 13.75 & $19.68^{\star \star}$ & 3.68 & $26.45^{\star \star}$ \\
\hline x G429 & 2.76 & 138.85 & 2.78 & -7.26 & -27.15 & -7.26 & -2.02 & -0.28 & $5.45^{\star}$ \\
\hline x Sakha 3 & $-25.28^{\star *}$ & -1.37 & $-8.33^{\star}$ & $-33.33^{* \star}$ & -1.16 & -6.67 & $-15.72^{\star \star}$ & -1.55 & $-6.20^{\star}$ \\
\hline$x$ R.V $V_{323}$ & $-22.78^{\star *}$ & -22.26 & $-21.99^{\star \star}$ & $-23.86^{\star \star}$ & -2.84 & $-17.06^{\star \star}$ & 0.39 & 0.04 & $13.01^{\star \star}$ \\
\hline Santamora x G429 (C5) & $-14.51^{*}$ & $\mid-0.82$ & 4.86 & $-16.07^{\star \star}$ & -0.58 & $17.50^{\star}$ & $-10.48^{\star \star}$ & -0.85 & 2.18 \\
\hline x Sakha3 & $-16.67^{\star}$ & -41.00 & $-16.67^{\star}$ & \begin{tabular}{|l|}
-14.12 \\
\end{tabular} & -29.24 & -14.12 & -7.53 & -1.56 & -2.85 \\
\hline x R.V V $_{323}$ & -3.75 & -0.24 & $16.67^{*}$ & 2.09 & 0.08 & $29.00^{\star \star}$ & $13.47^{\star \star}$ & 0.82 & $35.75^{\star *}$ \\
\hline Giza429 x Sakha3 & -4.88 & -0.26 & $16.67^{\star}$ & $-11.61^{\star}$ & -0.41 & $23.75^{\star \star}$ & $6.22^{\star \star}$ & 0.36 & $28.14^{\star \star}$ \\
\hline x R.V $V_{323}$ & 9.89 & 2.62 & 33.25 & -5.04 & -0.47 & $-21.43^{\star \star}$ & $-15.35^{\star \star}$ & -4.53 & $7.26^{\star \star}$ \\
\hline Sakha3 x R.V V 323 & & 0.09 & $33.33^{\star \star}$ & & 0.26 & $40.00^{\star \star}$ & & 0.23 & $35.32^{\star \star}$ \\
\hline \begin{tabular}{|r|} 
LSD 0.05 \\
0.01 \\
\end{tabular} & & & & & & & & & $\begin{array}{l}2.257 \\
3.456\end{array}$ \\
\hline
\end{tabular}

${ }^{\star}$ and $^{\star *}$ significant at 0.05 and 0.01 levels of probability, respectively.

Table 8: Cont.

\begin{tabular}{|c|c|c|c|c|c|c|c|c|c|c|}
\hline \multirow{2}{*}{\multicolumn{2}{|c|}{ Crosses }} & \multicolumn{3}{|c|}{ Plant height } & \multicolumn{3}{|c|}{ No. of branches plant ${ }^{-1}$} & \multicolumn{3}{|c|}{ No. of pods plant ${ }^{-1}$} \\
\hline & & MP & PR & BP & MP & PR & BP & MP & PR & BP \\
\hline Giza40 x Santamora & (C1) & $6.42^{\star \star}$ & 1.52 & 2.10 & $40.02^{\star \star}$ & 4.22 & $27.89^{*}$ & $42.44^{\star \star}$ & 23.18 & $39.88^{\star \star}$ \\
\hline x G429 & (C2) & $13.99^{\star \star}$ & 11.97 & $12.68^{\star \star}$ & 20.62 & 10.00 & 18.18 & $6.99^{\star}$ & 1.06 & 0.37 \\
\hline & (C3) & $.58^{\star \star}$ & $|-0.92|$ & $-14.58^{\star \star}$ & 5.56 & 4.29 & & & 6.54 & $27.87^{\star \star}$ \\
\hline$x$ R.V $V_{323}$ & (C) & 18.6 & \begin{tabular}{|l|}
0.97 \\
\end{tabular} & \begin{tabular}{|l|}
-0.41 \\
\end{tabular} & .05 & -0.0 & & & 14.04 & 8.47 \\
\hline antamora x G429 & (C! & \begin{tabular}{|l|}
-0.32 \\
\end{tabular} & $\mid-0.06$ & $-5.42^{\star \star}$ & $25.04^{\star}$ & 2.17 & 2.12 & 2.1 & 0.45 & -2.49 \\
\hline & & & -0.87 & $-7.17^{\star \star}$ & 16.33 & 1.99 & & $19.06^{\star \star}$ & 2.68 & 11.14 \\
\hline$x R \cdot V_{323}$ & & $22.58^{\star \star}$ & \begin{tabular}{|l|}
0.97 \\
\end{tabular} & -0.49 & 10.00 & 0.74 & -3.1 & $11.52^{*}$ & 4.64 & 8.81 \\
\hline iza429 x Sakha3 & (C8) & $3.38^{*}$ & 0.36 & -5.47 & $58.56^{\star \star}$ & 17.45 & $53.41^{\star \star}$ & $49.08^{\star \star}$ & 4.14 & $33.28^{\star \star}$ \\
\hline x R.V & (C9 & 3 & 0.22 & -1.60 & 22.22* & 10.63 & 15.99 & $32.97^{\star \star}$ & 4.03 & 9.90 \\
\hline Sakha3 x R.V ${ }_{323}$ & (C10) & $21.08^{\star \star}$ & 0.75 & $-8.07^{\star \star}$ & $86.46^{\star *}$ & 13.30 & 76.7 & 53.8 & 5.29 & $46.97^{\star \star}$ \\
\hline $\begin{array}{r}\text { LSD } 0.05 \\
0.01\end{array}$ & & \begin{tabular}{|l|}
4.406 \\
5.944
\end{tabular} & & $\begin{array}{l}5.088 \\
6.864\end{array}$ & \begin{tabular}{|l|}
0.817 \\
1.102 \\
\end{tabular} & & & & & $\begin{array}{l}3.361 \\
4.535\end{array}$ \\
\hline
\end{tabular}

*and ${ }^{\star *}$ significant at 0.05 and 0.01 levels of probability, respectively.

Table 8: Cont.

\begin{tabular}{|c|c|c|c|c|c|c|c|c|c|}
\hline \multirow{2}{*}{ Crosses } & \multicolumn{3}{|c|}{ No. of seeds plant ${ }^{-1}$} & \multicolumn{3}{|c|}{ Seed yield plant $^{-1}$} & \multicolumn{3}{|c|}{ 100-seed weight } \\
\hline & M.P & P.R & B.P & M.P & P.R & B.P & M.P & P.R & B.P \\
\hline Giza40 x Santamora (C1) & $37.28^{\star \star}$ & 109.15 & $36.81^{\star *}$ & $23.96^{\star \star}$ & 1.69 & $8.53^{\star \star}$ & $-8.67^{\star \star}$ & -0.61 & $-19.97^{\star \star}$ \\
\hline x G429 (C2) & 3.00 & 1.06 & 0.17 & -4.75 & -2.15 & -6.81 & $-7.65^{\star \star}$ & -10.85 & $-8.29^{\star \star}$ \\
\hline x Sakha 3 & -2.25 & -1.05 & -4.30 & $\left|-12.59^{\star \star}\right|$ & -1.75 & $-18.47^{\star \star}$ & $-9.97^{\star \star}$ & -1.07 & $-17.65^{\star \star}$ \\
\hline$x$ R.V $V_{323}$ & -0.21 & -0.12 & -1.87 & $16.96^{\star \star}$ & 0.74 & -4.78 & $21.71^{\star \star}$ & 0.73 & $-6.24^{\star \star}$ \\
\hline Santamora x G429 & $8.37^{\star}$ & 2.65 & $5.05^{\star}$ & -1.45 & -0.12 & $-12.05^{\star \star}$ & $-10.99^{\star *}$ & -0.74 & $-22.48^{\star *}$ \\
\hline x Sakha3 & $10.94^{\star \star}$ & 6.10 & $8.99^{*}$ & $10.79^{\star \star}$ & 1.52 & 3.46 & 0.05 & 0.01 & $-4.60^{\star \star}$ \\
\hline x R.V $V_{323}$ & 2.55 & 1.26 & 0.51 & $13.74^{\star \star}$ & 0.38 & $-16.29^{\star \star}$ & $14.78^{\star \star}$ & 0.35 & $-19.27^{\star \star}$ \\
\hline Giza429 x Sakha3 & $58.91^{\star \star}$ & 11.90 & $51.41^{\star \star}$ & $57.53^{\star \star}$ & 11.50 & $50.03^{\star \star}$ & $1.46^{*}$ & 0.15 & $-7.78^{\star \star}$ \\
\hline x R.V $\mathbf{V}_{323}$ & $22.12^{\star \star}$ & 18.56 & $14.99^{\star \star}$ & $42.07^{\star \star}$ & 1.80 & $21.21^{\star \star}$ & $19.04^{\star \star}$ & 1.04 & $5.41^{\star}$ \\
\hline Sakha3 x R.V $V_{323}$ & 48.13 & 7.22 & $42.69^{\star \star}$ & 45.35 & 1.79 & $30.47^{\star *}$ & $23.96^{\star \star}$ & 0.58 & $-17.53^{\star \star}$ \\
\hline LSD 0.05 & 6.095 & & 6.819 & 4.274 & & 4.934 & 2.435 & & 2.812 \\
\hline
\end{tabular}

*and ${ }^{\star \star}$ significant at 0.05 and 0.01 levels of probability, respectively 
Salwa M. Mostafa, et al.,

Table 8: Cont.

\begin{tabular}{|c|c|c|c|c|c|c|c|}
\hline \multicolumn{2}{|l|}{ Crosses } & \multicolumn{3}{|c|}{ Crude protein content } & \multicolumn{3}{|c|}{ Carbohydrate \% } \\
\hline & & M.P & $P, R$ & B.P & $\mathbf{M}, \mathbf{P}$ & P.R & $\mathbf{B}, \mathbf{P}$ \\
\hline \multicolumn{2}{|c|}{ Giza40 x Santamora (C1) } & -2.28 & -0.39 & $-7.71^{\star \star}$ & $7.63^{\star \star}$ & 1.97 & $3.61^{* *}$ \\
\hline x G429 & (C2) & $12.69^{\star \star}$ & 43.60 & $12.37^{\star \star}$ & -0.50 & -0.39 & -1.77 \\
\hline x Sakha 3 & (C3) & $10.10^{\star \star}$ & 3.51 & $7.03^{\star \star}$ & $-5.95^{\star \star}$ & -6.35 & $-6.82^{\star \star}$ \\
\hline x R.V V $_{323}$ & (C4) & $19.89^{\star \star}$ & 3.10 & $12.66^{\star *}$ & $-5.46^{\star \star}$ & -3.25 & $-7.02^{\star \star}$ \\
\hline Santamora x G429 & (C5) & $-8.93^{\star \star}$ & -1.45 & $-14.22^{\star \star}$ & 3.90 & 0.75 & -1.21 \\
\hline x Sakha3 & (C6) & $9.77^{\star \star}$ & 3.24 & $6.56^{\star \star}$ & $-4.07^{\star \star}$ & -0.85 & $-8.47^{\star \star}$ \\
\hline x R.V . $_{323}$ & (C7) & $-5.71^{*}$ & -10.61 & $-6.22^{\star \star}$ & $5.59^{\star \star}$ & 2.54 & $3.32^{\star \star}$ \\
\hline Giza429 x Sakha3 & (C8) & $11.03^{\star \star}$ & 3.48 & $7.62^{\star \star}$ & $-2.06^{\star}$ & -5.85 & $-2.40^{*}$ \\
\hline x R.V 323 & (C9) & 0.94 & 0.14 & -2.52 & -0.54 & -0.18 & $-3.76^{\star \star}$ \\
\hline Sakha3 x R.V V $_{323}$ & (C10) & 1.53 & 0.43 & -2.89 & $-1.74^{\star}$ & -0.67 & $-3.42^{\star \star}$ \\
\hline $\begin{array}{r}\text { LSD } 0.05 \\
0.01\end{array}$ & & $\begin{array}{l}0.974 \\
1.314\end{array}$ & & $\begin{array}{l}1.124 \\
1.516\end{array}$ & $\begin{array}{l}0.999 \\
1.348\end{array}$ & & $\begin{array}{l}1.153 \\
1.556\end{array}$ \\
\hline
\end{tabular}

*and ${ }^{\star \star}$ significant at0.05 and 0.01 levels of probability, respectively

highly significant mid-parental heterosis in positive direction (favorable) for plant height due to over-dominance, while the crosses; Giza $40 \times$ R. $V_{323}$, Santa Mora $x \times$ R. $V_{323}$, Giza429 $x$ Sakha3 and Sakha3 $x$ R. $V_{323}$ expressed mid- parental heterosis for the trait in view due to partialdominance. For No, of branches plant ${ }^{-1}$, the crosses; Giza40 x Santamora, Santamora x Giza429,Giza429 x Sakha3, Giza429 x R.V $V_{323}$ and Sakha3 $\times$ R. $V_{323}$ had significant mid-parental heterosis due to over-dominance (PR>+1). For No. of pods plant ${ }^{-1}$, all crosses, except the cross; Santamora x Giza429expressed significant and /or highly significant midparental heterosis due to overdominance.

For No. of seeds plant ${ }^{-1}$, the crosses; Giza40 x Santamora, Santamora $x$ Giza429, Santamora x Sakha3, Giza429 x Sakha3, Giza429 x R.V H23 $_{32}$ and Sakha3 $x$ R. $V_{323}$ exposed significant mid- parental heterosis due to over- dominance. For seed yield plant ${ }^{-1}$ all crosses, except for Giza40 x Giza429, Giza40 x Sakha3 and Santamora $x$ Giza429 had highly significant mid- parental heterosis due to over- dominance in the crosses; Giza40 x Santamora, Santamora x Sakha3, Giza429 x Sakha3 Giza429 x Sakha3, Giza429 $x$ R.V $V_{323}$ and Sakha3 $\times$ R.V V $_{323}$, the heterotic effects in the rest crosses were affected by partial dominance.

For 100-seed weight, the crosses; Giza $40 \times$ R.V V23 $_{32}$, Santamora $\times$ R.V $_{323}$, Giza429 $x$ Sakha3 and Sakha3 x R.V V $_{323}$ showed significant mid-parental heterosis due to partial-dominance, the cross; Giza429 xR. $V_{323}$ was the only one which heterotic effects were due to overdominance .For crude protein content, the crosses; Giza40 x Giza429, Giza40 x Sakha3, Giza $40 \times$ R.V $_{323}$ Santamora $x$ Sakha3 and had highly significant midparental heterosis a result of overdominance as potence ratio pointed out and for carbohydrate\% where the crosses; Giza40 X Santamora, Santamora X R. $\mathbf{V}_{323}$ expressed highly significant mid-parental heterosis due to over-dominance.

Better-parent heterosis as the results presented in Table 8 had significant and highly significant in the cross; Giza40 $x$ Santa Mora for No. of branches plant ${ }^{-1}$, No. of podsplant ${ }^{-1}$, No. of seedsplant ${ }^{-1}$ 
,seed yield plant ${ }^{-1}$ and carbohydrate $\%$; in the cross; Giza429 x Sakha3 for No of branches plant $^{-1}$, No. of podsplant ${ }^{-1}$, No. of seedsplant ${ }^{-1}$, seed yield plant $^{-1}$ and crude protein content; in the cross; Giza40 x Sakha3 for chocolate spot disease reaction, flowering date, No. of pods plant $^{-1}$ and crude protein content; in the cross; Giza 429 x R. V $_{323}$ for rust disease reaction, No. of pods plant ${ }^{-1}$, No. of seeds plant $^{-1}$, seed yield plant 1 and100-seed weight; in the cross; Sakha3 $x$ R. $V_{323}$ for No. of branches plant $^{-1}$, No. of pods plant ${ }^{-1}$, No. of seeds plant $^{-1}$ and seed yield plant ${ }^{-1}$; in the cross; Giza $40 \times$ R.V $V_{323}$ for chocolate spot and rust disease reactions and crude protein content and in the cross; Santamora $x$ Sakha3 for chocolate spot disease reaction, No. of seeds plant ${ }^{-1}$ and crude protein content.

It should be noticed that, these is an approximately accordance between specific combining ability effects in the present study and heterosis over betterparent, which pointed out the important role of non-additive gene effects in controlling the inheritance of these traits in question, and this may confirm the obtained results mentioned before.

These data suggest that heterotic effects for seed yield plant ${ }^{-1}$ were associated with other yield components, in several crosses, such as 100-seed weight and No. of pods plant ${ }^{-1}$. Moreover, various cross combinations exhibited different degrees of crosses superiority in some traits based on the genes in parental combinations that may contribute directly, or indirectly, to the expression of these traits. In addition, the heterosis estimates, compared to either MP or BP, for seed yield plant ${ }^{-1}$ and its major yield components traits indicated that there was sufficient genetic variability among the assessed parents to favor efficient breeding for these traits. Therefore, the progenies of these crosses could be used in the segregating generations to regenerate new genotypes characterized by high yielding potentiality and high protein content and resistance to foliar diseases. These results are in good agreement with those reported by Darwish et al (2005), Attia et al., (2006), Farag (2007), El-Hady et al., (2008), Farag and Afiah (2012), Ahmed (2016), Abdalla et al., (2017) and AbouZaid et al., (2018).

The difference in percent heterosis might be due to genetic differences of the parents used and or non- allelic interaction which can either increase or decrease the expression of heterosis (Cress, 1966). Aabdalla (1977) reported that, heterosis was very pronounced in $F_{1}$ especially among widely divergent materials but was less manifested in hybrids between local varieties.

Heterosis over better parent is more important than heterosis over mid-parent from the breeder point of view, especially if the heterotic effects are due to overdominance $(P>+1$ or $<-1)$, the case which allow the breeder to searched out the transgressive segregates in the segregating generations.

In conclusion, the results revealed that several crosses are highly promising to breed new faba bean genotypes possessing genetic factors for resistance to chocolate spot and rust foliar diseases, earliness and high yielding ability.

\section{REFERENCES}

Abdalla, M.M.F. (1977). Performance of $F_{1}$ and $F_{2}$ hybrids of Vicia faba L. Egypt. J. Genet. Cytol., 6:106-121.

Abdalla, M.M.F, M.M. Shafik, Sabah M Attia and Hend A. Ghannam (2017). Heterosis, GCA and SCA effects of diallel-cross among six faba bean Vicia faba L.) genotypes. Asian Res. J. Agric., 4(4):1-10. 
Abo El-Zahab, A.A, S.A. Khalil, N.M. AboZeid, H.H. El-Hinnawy and M.M. ElHady (1994). Faba bean (Vicia faba L.) resistance to chocolate spot disease caused by Botrytis fabae Sard. I. Recisrocal 130 differences and combining ability among faba bean crosses. Proc. 6th Conf. Agron., AlAzhar Univ., 2: 651- 673.

Abou-Zaid Gehan, G.A., M. M. Salwa, R.A. El-Refaey and A. M. Mohamed (2018). Estimation of combining ability and hetrosis Via Half diallel cross in faba bean (Vicia faba L.) for yield, its components and seed quality. J. Plant Production, Mansoura Univ., 8(11): 1191 - 1198.

Ahmad, M.S.H. (2016). Studies on genetic variability, heritability and genetic advance in segregating generations of faba bean (Vicia faba L.). Middle East J. Agric. Res., 5(1):82-89.

Attia, Sabah. $M$ and Manal M. Salem (2006). Analysis of yield and its components using diallel matings among five parents of faba bean. Egypt. J. Plant Breed., 10:1-12.

AOAC (2006). Official methods of analysis of association of official analyical chemists international, 18th Edition, published by AOAC international Maryland, USA.

Bernier, C.C., S.B. Hanounik, M.M. Hussein and H.A. Mohamed (1984). Field manual of common faba bean diseases in Nile Valley. Information Bulletin No. 3. ICARDA, P.O Box 5466, Aleppo, Syria.

Cress C.E. (1966). Heterosis of the hybrid related to gene frequency differences between two populations. Genetics., 53: 269-274.

Darwish, D.S., M.M.F. Abdalla and E.A.A. El-Emam (2005). Investigations on faba bean (Vicia faba L.) .19-diallel and triallel matings using five parents. Proceed. Fourth PI. Breed. Conf.
March 5, (Ismailia) Egypt. J. Plant Breed., 9(1):197-208.

El-Bramawy, M.A.S. and M.A.M. Osman (2012). Diallel crosses of genetic enhancement for seed yield components and resistance to leaf miner and aphid infestations of Vicia faba L. Int. J. Agron. Agric. Res. (IJAAR), 2 (2): 8-21

El-Hady, M.M, Sabah M. Attia, Ola A.M. ElGalaly and Manal M. Salem (2006). Heterosis and combining ability analysis of some faba bean genotypes. J. Agric. Res. Tanta Univ., 32(1): 134-148.

El-Hady, M.M., A.M. Rizk, M.M. Omran and S.B. Regheb (2007). Genetic behavior of some faba bean (Vacia faba L) genotypes and its crosses. Ann. Agric. Sci., Moshtoher, 45(1): 49-60.

El-Hady, M.M., Sabah M. Attia, E. A.A. ElEmam, A.A.M. Ashrei and E.M. Rabie (2008). Diallel mating among eight parents of faba bean (Vacia faba $L$ ) and performance of $F_{1}$ and $F_{2}$. Egypt. J. of Appl. Sci., 23(5): 95-114.

El-Hosary, A.A., S. Shokr, A.A.A. ElHalim, S.M. Nasr and A.M.A. El-Aziz (1997). Heterosis and combining ability in faba bean (Vicia faba L.), Egypt. J. of Agric. Res., 75: 811-826.

Farag, S.T. (2007). Relative importance of genetic variance for improving broad bean (Vacia faba L.). Proceed. Fifth PI. Breed. Conf. May 27 (Giza) Egypt. J. Plant Breed., 11(1): 301-315.

Farag, H.I.A. and S.A. Afiah (2012). Analysis of gene action in diallel crosses among some faba bean (Vacia faba L.) genotypes under Maryout conditions. Annals of Agric. Sci., 57(1): 37- 46.

Foolad, M.R. and A. Bassiri (1983). Estimates of combining ability, reciprocal effects and heterosis for yield and yield components in a 
common bean diallel cross. $J$. of Agric. Sci., 100: 103-108.

Ghareeb, Zeinab E. and A.G. Helal (2014). Diallel analysis and separation of genetic variance components in eight faba bean genotypes. Ann of Agric. Sci., 59 (1):147-154.

Griffing J.B. (1956). Concept of general and specific combining ability in relation to diallel crossing systems. Aust. J. Biol. Sci., 9:463-493

Haciseferogullari, H., I. Geaer, Y. Bahtiyarca and H.O. Menges (2003). Determination of some chemical and physical properties of Sakiz faba bean (Vicia faba L. Var major). J. Food Eng., 60: 476- 479 .

Ibrahim, H.M. (2012). Heterosis, combining ability and components of genetic variance in faba bean (Vicia faba L.). Meteorology, environment and arid land agriculture. Science, 21(1): 35-50.

Lindemann, C. and R. Glover (2003). Nitrogen fixation by legumes, New Mexico State University, Mexico. Available at: www.cahe.nmus. edu/pubs/_a/a-129.pdf.
Mather, K. and J.L. Jinks (1971). Biometrical Genetics. $2^{\text {nd }}$ ed. Chapman and Hall Ltd., London, pp.382.

Rowlands, D. G. (1955). The problem of yield in field beans. Agric. Prog. 30: 137-147.

Salih, F. A and S. B. El-Hadallou (1986). The yield performance and proxy,ate analysis of twelve cultivars of faba bean grown at five sites in the northern region of the sudan. FABI, 15-52-58.

Suso, M.J. and M.T. Moreno (1999). Variation in out crossing rate and genetic structure on six cultivars of Vicia faba L. as affected by geographic location and year. Plant Breed., 118: 347-350.

Tewatia, B. S and A. S. Virk (1996). Nutritional potential of faba bean for improved productivity in ruminants/ AReview. FABIS, 38:2-11.

Wigan, L.G. (1944). Balance and potence in natural populations. J. Genet., 46:150-160.

Wynne, J.C., D.A. Every and P.W. Rice (1970). Combining ability in Arachis hypogeae L.G. Field performance of $F_{1}$ hybrids. Crop Sci., 10 (6): 713-715. 
تقدير قوة الهجين والقدرة على التألف لصفات مقاومة الأمراض الورقية، المحصول ومكوناته وصفات الجودة في الفول البلاي

سلوى محمد مصطفى(')، جيهان جلال عبدالغفار أبوزيد(')، شيماء فرج احمد كلبوش(')،

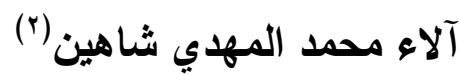

(1) قسم بحوث المحاصيل البقوليه - مع هد بحوث المحاصيل الحقلية، مركز البحوث الزراعية

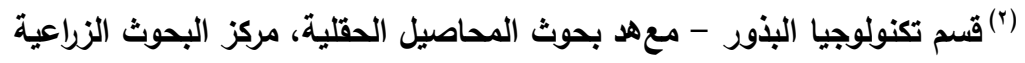

الملخص العربي

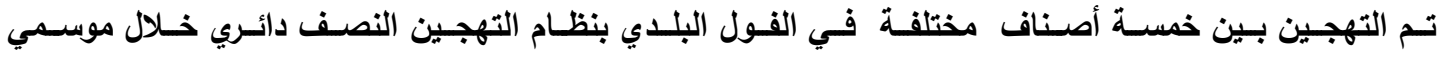

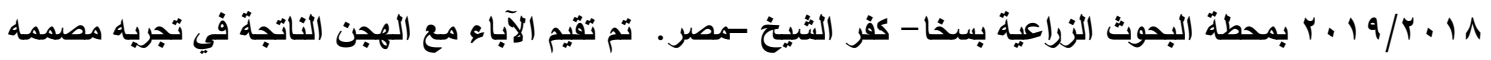

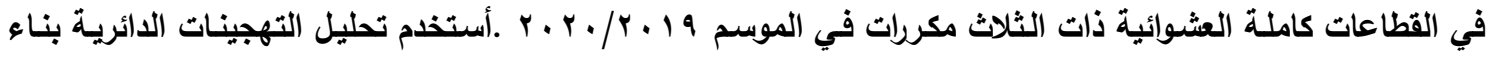

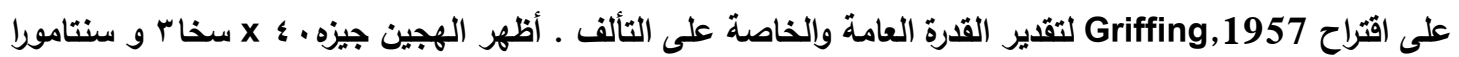

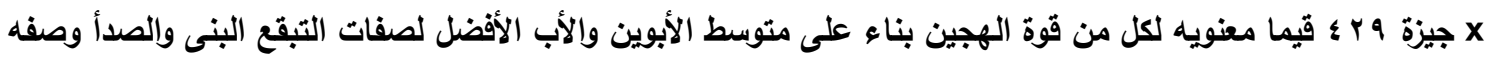

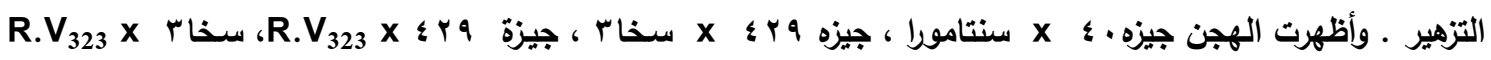

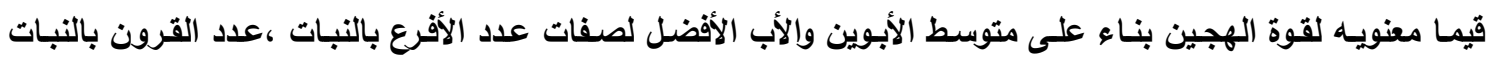

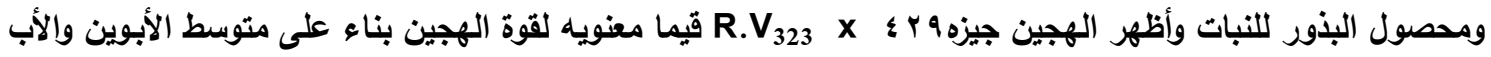

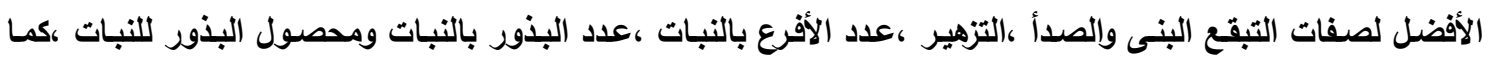

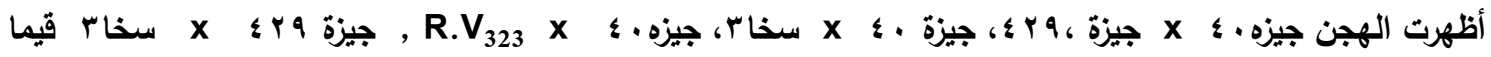

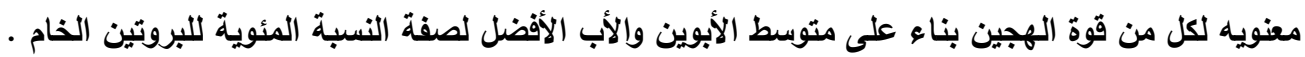

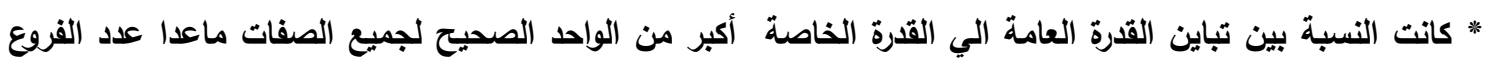

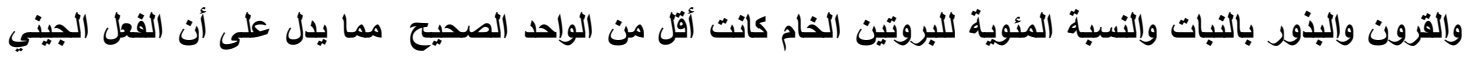

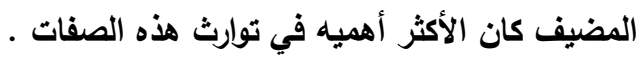

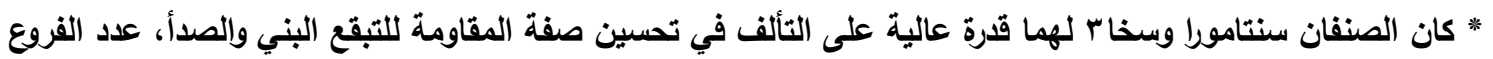

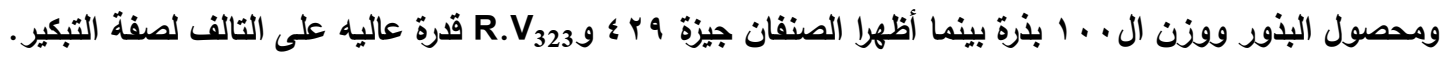

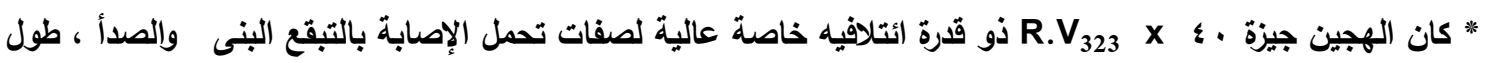

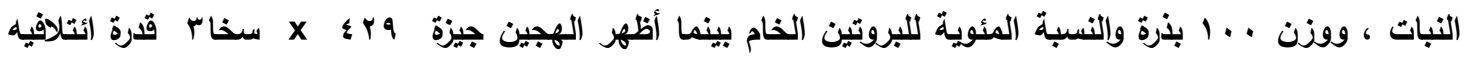

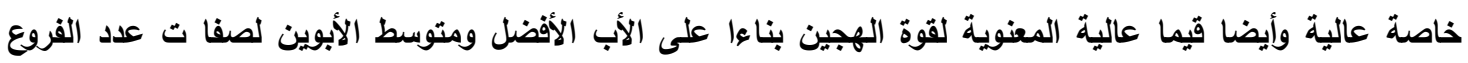

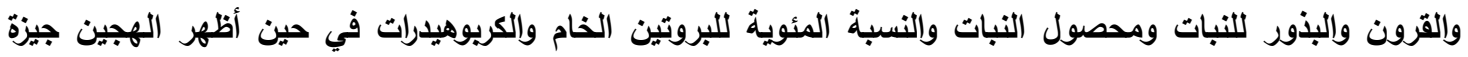
R.V323 x x a 9

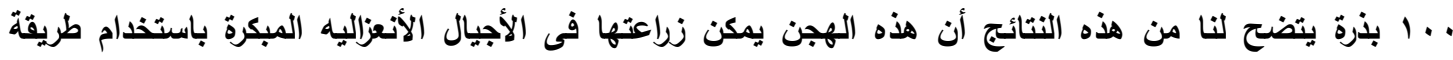
الانتخاب التجميعي لإنتاج سلالات عاليه في المحصول والمقاومة للأمراض.

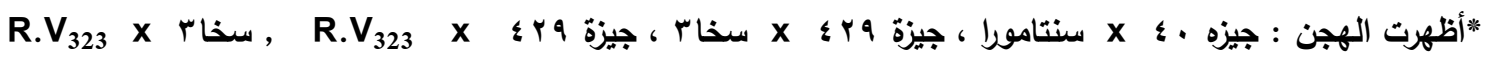

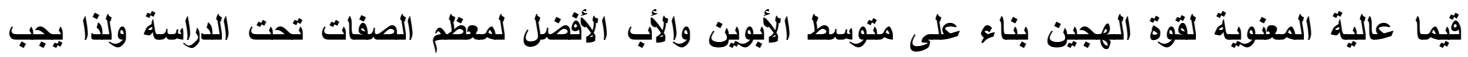

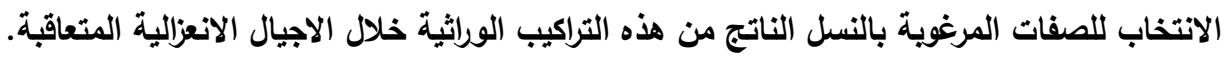

أ.د/ محمد سعد عبدالعاطى كلية الزراعة - جامعة كفر الثيخ، أ.د/ إبراهيم حسينى درويش كلية الزراعة - جامعة المنوفية 
$\underline{\text { Estimates of heterosis and combining abilaty for foliar diseases resistance, ........ }}$ 